\title{
Electronic and magnetic properties of $\mathrm{GaFeO}_{3}: \mathrm{Ab}$ initio calculations for varying $\mathrm{Fe} / \mathrm{Ga}$ ratio, inner cationic site disorder, and epitaxial strain
}

\author{
J. Atanelov* and P. Mohn \\ Institute of Applied Physics, Vienna University of Technology, Gußhausstraße 25-25a, 1040 Vienna, Austria
}

(Received 18 May 2015; published 11 September 2015)

\begin{abstract}
In this study we present $a b$ initio density-functional theory calculations on stoichiometric, cation-doped, and strained $\mathrm{GaFeO}_{3}$. We start with a detailed discussion of the origin of the antiferromagnetic (AFM) superexchange in stoichiometric $\mathrm{GaFeO}_{3}$ and give a molecular orbital description of the exchange mechanism derived from our calculations. In addition, we study the properties of the Fe-O-Fe bonds for different geometries to underline the angle and distance dependence of the AFM coupling as formulated in the Goodenough-Kanamori rules. We describe the AFM ground state of $\mathrm{GaFeO}_{3}$ as a result of two intrinsic $\mathrm{Fe}-\mathrm{O}$-Fe chains that meander through the crystal along the $c$ direction. The magnetocrystalline anisotropy energies are calculated for the stoichiometric phase with and without inner cationic site disorder, and the presence of a sublattice-dependent anisotropy is examined. Furthermore, we perform our studies of $\mathrm{Ga}_{2-x} \mathrm{Fe}_{x} \mathrm{O}_{3}$ for varying Fe concentrations $x(0.0 \leqslant x \leqslant$ 2.0) where at a value of $x=0.0$ and $x=2.0$ it transforms into the isomorphic $\varepsilon-\mathrm{Ga}_{2} \mathrm{O}_{3}$ and $\varepsilon-\mathrm{Fe}_{2} \mathrm{O}_{3}$ phases, respectively. The effect of strain was also studied. Incorporating dopants and applying strain to the simulation cell changes the intrinsic geometry and thus the magnetic properties of gallium ferrite.
\end{abstract}

DOI: 10.1103/PhysRevB.92.104408

PACS number(s): 75.85.+t, 74.62.Dh, 75.30.Et, 71.15.Mb

\section{INTRODUCTION}

$\mathrm{GaFeO}_{3}$ (GFO) is a multiferroic material showing both antiferromagnetic (AFM) and ferroelectric behavior in its ground state. Ferroelectricity and magnetism coexist in a single phase, leading to the so-called magnetoelectric effect (ME) [1-3]. Magnetic and electric properties couple in such a system. Consequently, the magnetic properties can be altered via an applied electric field and the inner electric properties, like polarization, can be changed by an external magnetic field. This makes GFO suitable for a wide range of applications in electronic devices and an interesting material for further investigations.

Most of the multiferroic materials have magnetic ordering temperatures below room temperature as well as an AFM ground state. A common method to increase the magnetic ordering temperature and to overcome the AFM ground state is the incorporation and exchange of atoms. A great number of recent experimental and computational studies examined the effect of excess Fe mainly focusing on a doping concentration of $0.9 \leqslant x \leqslant 1.4$ in $\mathrm{Ga}_{2-x} \mathrm{Fe}_{x} \mathrm{O}_{3}$. They report an increase of the ordering temperature and the total magnetic moment with increasing amount of $\mathrm{Fe}[1,4-7]$. Since both $\varepsilon-\mathrm{Ga}_{2} \mathrm{O}_{3}(\varepsilon-\mathrm{GO})$ and $\varepsilon-\mathrm{Fe}_{2} \mathrm{O}_{3}(\varepsilon-\mathrm{FO})$ are isomorphic structures of GFO [8,9], doping concentrations of $0.0 \leqslant x \leqslant 2.0$ can be investigated without facing any phase stability problems.

GFO is also known to exhibit inner cationic site disorder $[1,7,10,11]$ causing the total magnetic moment to be nonzero without changing the $\mathrm{Fe}$ concentration.

\footnotetext{
*ja@cms.tuwien.ac.at

Published by the American Physical Society under the terms of the Creative Commons Attribution 3.0 License. Further distribution of this work must maintain attribution to the author(s) and the published article's title, journal citation, and DOI.
}

The electric polarization is a main feature of GFO. Recent experimental and theoretical studies investigate its origin and a possible alteration $[3,5,12,13]$.

Another interesting property of GFO is its magnetocrystalline anisotropy energy (MAE). Experimental and computational studies $[1,14,15]$ reveal the $c$ axis as the magnetic easy axis and the $b$ axis as the magnetic hard axis of the system. In addition, Reddy et al. [6] have shown a sublattice-dependent MAE in GFO. Motivated by that we calculate the energetic difference and the contribution of the respective sublattices to the total MAE by including spin-orbit coupling in our calculations. This is done for pure $\mathrm{GaFeO}_{3}$ and for the case of inner cationic site disorder.

The application of strain is also known to possibly alter the electronic and magnetic properties of a system, due to stress induced changes in the cell geometry. Strain evoked changes in the AFM coupling strength are examined, stretching and compressing the simulation cell in the $a, b$, and $c$ directions while keeping the volume constant. This is done for a doping concentration of $0.9 \leqslant x \leqslant 1.4$ and the case of inner cationic site disorder.

GFO has a noncentrosymmetric crystal structure and belongs to the $\mathrm{Pc} 2{ }_{1} \mathrm{n}$ (No. 33) space group. The unit cell is orthorhombic with experimentally determined lattice parameters of $a=8.735 \AA, b=9.383 \AA$, and $c=5.077 \AA$ [11]. Formally, iron and gallium are both in a $3^{+}$state, whereas oxygen is in a $2^{-}$state. The magnetic moment is located on the Fe atoms, showing a magnetic moment of about $4 \mu_{B}$ /atom $[1,11,15]$ making GFO an AFM semiconductor. GFO has four cationic sublattices indexed by 1 and 2 for $\mathrm{Ga}$ and $\mathrm{Fe}$, respectively (Fig. 1). Fe atoms on the $\mathrm{Fe} 1$ and $\mathrm{Fe} 2$ sites couple antiferromagnetically and as well as Ga2 sites are surrounded by an oxygen octahedron. Ga1 is located inside an oxygen tetrahedron.

Performing ab initio DFT calculations of the electronic and magnetic structure we examine the effects of strain, cation doping, and inner cationic site disorder on the AFM coupling strength. Our calculations yield a better understanding of the 


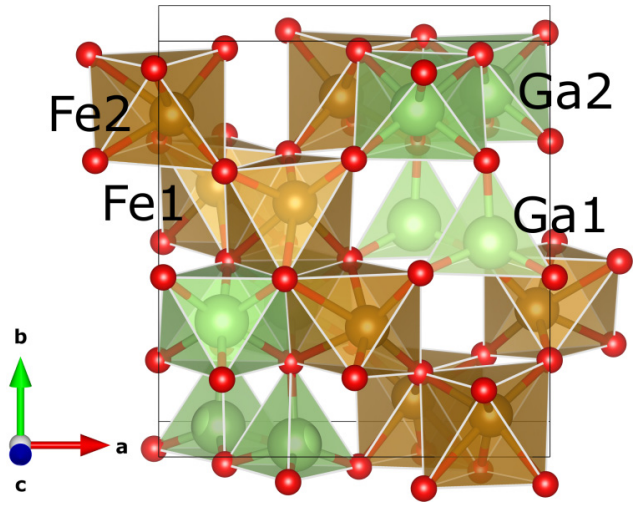

FIG. 1. (Color online) Cell structure of pure GFO. The Ga1, Ga2, $\mathrm{Fe} 1$, and $\mathrm{Fe} 2$ sublattices are indicated.

magnetic properties of GFO and therefore may support the possibilities of tailoring the electronic and magnetic properties of GFO for a future application in electronic devices.

\section{COMPUTATIONAL DETAILS AND METHODS}

All calculations were carried out with the Vienna Ab initio Simulation Package (VASP) [16-21], which uses projector augmented wave (PAW) pseudopotentials [22] to describe the potential between the ions.

The simulations were carried out on an 8-f.u. (formula units) unit cell containing 40 atoms. To provide a reliable description of the effects of electronic correlation the calculations were performed using the GGA $+\mathrm{U}$ formalism [23] and Heyd-Scuseria-Ernzerhof (HSE) hybrid functionals [24-27]. The GGA+U implementation uses the Dudarev formalism. The effective on site Coulomb and exchange parameters were set to $U=5 \mathrm{eV}$ and $J=1 \mathrm{eV}$, yielding reliable results for the magnetic moments and the cell parameters as compared to experiment.

In the case of doped and strained GFO the simulation cell was relaxed within the GGA $+\mathrm{U}$ approximation until all force components were smaller than $0.01 \mathrm{eV} / \AA$. The atomic positions, the shape, and the volume of the simulation cell were allowed to change during relaxation. Plane waves with an energy up to $550 \mathrm{eV}$ were included in the basis set in order to avoid Pulay forces. The Brillouin-Zone integration was performed on a $6 \times 6 \times 6 \Gamma$ centered $k$ mesh with a Gaussian smearing of $0.05 \mathrm{eV}$. Total energies were converged better than $1 \times 10^{-6} \mathrm{eV}$.
Calculating the MAE the spin-orbit coupling was included to the calculations. Since spin-orbit-related properties are influenced by geometry we compared the MAE for simulation cells relaxed within the GGA $+\mathrm{U}$ or with the more realistic but computationally more elaborate HSE functional.

Using the GGA+U approximation the simulation cell was relaxed until all force components were smaller than $0.001 \mathrm{eV} / \AA$. For the HSE functional the short-range/longrange splitting parameter was determined to be $\omega=0.7$ for an optimal description of the experimental band gap and crystal parameters. To account for the vastly increased computation time the $k$-space integration was performed on a smaller $4 \times 4 \times 4 \Gamma$ centered $k$ mesh. The simulation cell was relaxed until all force components were smaller than $0.01 \mathrm{eV} / \AA$ (see the Supplemental Material SM1 [28] for HSE-relaxed cell structure).

To quantify the AFM superexchange of the systems studied in this work we introduce the AFM coupling strength as a main parameter. In pristine GFO the AFM coupling strength is defined as the energy difference between the AFM ground state and a hypothetical ferromagnetic (FM) state. Forcing the system into a FM state breaks the ground-state AFM couplings. The corresponding energy difference is taken as a reference to measure the AFM coupling strength of the respective investigated system. Doping GFO can induce a ground state with a total magnetic moment unequal to zero. The AFM coupling strength is then defined by the energy difference between the actual magnetic ground state of the doped systems and again a hypothetical ferromagnetic state.

Table I lists the cell parameters, band gaps, and AFM coupling strengths of $\varepsilon$-GO, GFO, and $\varepsilon$-FO in the GGA+U and HSE calculation schemes, respectively.

\section{MAGNETIC PROPERTIES OF $\mathrm{GaFeO}_{3}$}

\section{A. AFM superexchange in $\mathrm{GaFeO}_{3}$}

The AFM ground state in GFO originates from the magnetic superexchange mediated via the Fe-O-Fe bonds. This AFM superexchange leads to a layerlike magnetic ordering. The direction of the magnetic moments of the Fe atoms occupying $\mathrm{Fe} 1$ sites are opposite to those located at the Fe2 sites.

If we apply a fully ionic picture $\mathrm{Fe}$ is in a $3^{+}$state and is left with five valence electrons in its $3 d$ shell which would result in a magnetic moment of $5 \mu_{B}$. However, measurements and calculations show a local magnetic moment of about $4 \mu_{B}$.

The calculated total density of states (DOS) in Fig. 2(a) (black line) shows the maximum of the $\mathrm{O} p$ states (blue

TABLE I. Experimental and calculated cell parameters of $\mathrm{GaFeO}_{3}(\mathrm{GFO}), \varepsilon-\mathrm{Fe}_{2} \mathrm{O}_{3}\left(\varepsilon\right.$-FO), and $\varepsilon-\mathrm{Ga}_{2} \mathrm{O}_{3}(\varepsilon-\mathrm{GO})$ in the AFM ground state. The magnetic stabilization energy $\Delta E$ is given per 40-atom simulation cell.

\begin{tabular}{|c|c|c|c|}
\hline & GFO & $\varepsilon-\mathrm{FO}$ & $\varepsilon-\mathrm{GO}$ \\
\hline Cell parameter a|b|c (HSE) $(\AA)$ & $8.77|9.44| 5.09$ & $8.82|9.55| 5.07$ & $8.72|9.33| 5.08$ \\
\hline Cell parameter $\mathrm{a}|\mathrm{b}| \mathrm{c}(\mathrm{GGA}+\mathrm{U})(\AA)$ & $8.82|9.50| 5.14$ & $8.85|9.56| 5.12$ & $8.81|9.42| 5.13$ \\
\hline Cell parameter $\mathrm{a}|\mathrm{b}| \mathrm{c}$ exp. $(\AA)$ & $8.74|9.38| 5.08[11]$ & $8.79|9.44| 5.10[8]$ & $8.71|9.3| 5.03[9]$ \\
\hline Band gap direct up|down (HSE) (eV) & 2.68 & $2.19 \mid 2.29$ & 3.11 \\
\hline Band gap direct up|down $(\mathrm{GGA}+\mathrm{U})(\mathrm{eV})$ & 2.43 & $1.91 \mid 2.16$ & 2.05 \\
\hline Band gap exp. up|down (eV) & $2.7-3.0[29,30]$ & - & - \\
\hline$\Delta E[A F M-F M](\mathrm{eV})$ & -1.47 & -4.94 & - \\
\hline
\end{tabular}




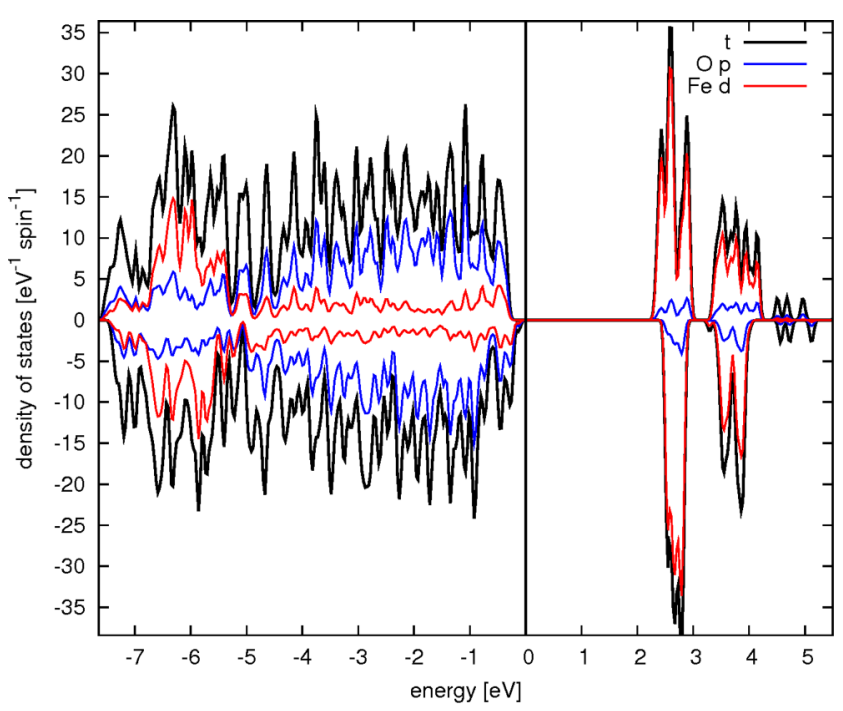

(a)

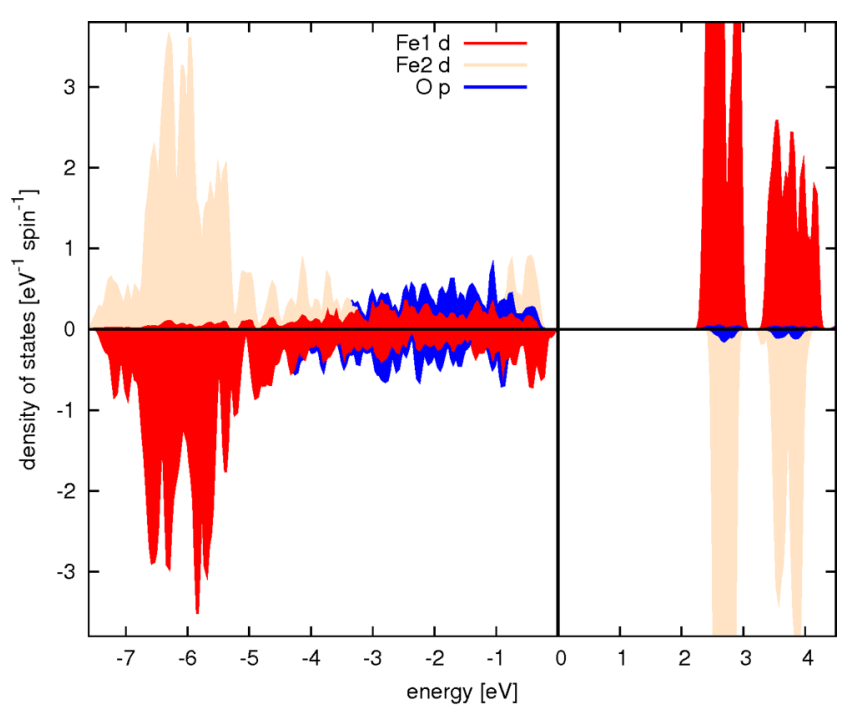

(b)

FIG. 2. (Color online) Panel (a) shows the total DOS (black), the overall Fe $d$ states (red), and the O $p$ states (blue) of pure GFO. Panel (b) shows the projected DOS of an Fe1-O-Fe2 complex. Plotted are the corresponding Fe1 (red area) and Fe2 (yellow area) $d$ states and the $\mathrm{O} p$ state (blue area). All DOS are calculated within the HSE approximation.

line) located in the upper part of the valence band near the Fermi level (all DOS are calculated within the HSE approximation). The barycenter of the Fe $3 d$ states (red line) is shifted downwards to lower energies. Both $\mathrm{O} p$ and $\mathrm{Fe} d$ states make up the majority of the total DOS near the Fermi level. Figure 2(b) shows the projected DOS of an Fe1-O-Fe2 complex inside the GFO simulation cell. There is a negative magnetic moment for iron on the Fe1 (red area) site and a positive magnetic moment for the iron on the Fe2 (yellow area) site. The $\mathrm{Fe} 1$ and $\mathrm{Fe} 2$ atom are antiferromagnetically coupled via an intermediate $\mathrm{O}$ atom (blue). The overlap of the oxygen and the iron states result in the DOS between $-5 \mathrm{eV}$ and the Fermi energy. The superexchange mechanism takes place in this particular energy range. Since the superexchange is the same for Fe1 and Fe 2 we now concentrate on the Fe1 states [Fig. 2(b)]. Integrating the spin-down part of the Fe DOS up to the Fermi energy (red area) yields five spin-down electrons. However, the integration over the corresponding spin-up states gives one electron, bringing the total magnetic moment on $\mathrm{Fe}$ down to the observed $4 \mu_{B}$.

The corresponding schematic molecular orbital (MO) diagram shown in Fig. 3 describes the AFM superexchange as it appears in an electronic band picture and in the corresponding schematic DOS. Oxygen forms a bond with each $\mathrm{Fe}$ atom mediating the observed AFM superexchange.

We start with an $\mathrm{Fe} 2^{+}$state with six $\mathrm{Fe} 3 d$ electrons. The spin-up electron of $\mathrm{Fe} 1$ forms a MO state with an $\mathrm{O} p$ electron. This covalent interaction not only leads to an increase of the Fe oxidation number towards $3^{+}$but also moves an Fe spin-up state below the Fermi energy and thus reduces the magnetic moment to the observed $4 \mu_{B}$. The same mechanism occurs on the $\mathrm{Fe} 2$ atom.

This mechanism is named "virtual electron transfer" and has been formulated in the Goodenough-Kanamori (GK) rules [31-34] where they state "The net spin of the cation orbital is not changed by addition of a covalent component but the covalent component extends the cation wavefunction out over the anions to give an orbital overlap for the superexchange electron transfer" [35].

\section{B. Distance and angle dependence of the AFM superexchange}

To investigate the angle and distance dependence of the AFM superexchange a single Fe1-O-Fe2 complex was left inside the simulation cell (see Fig. 4). Six of the eight $\mathrm{Fe}$ atoms are therefore exchanged by addtional $\mathrm{Ga}$ atoms. Interactions with neighboring Fe-O-Fe complexes can thereby be neglected, revealing the unaffected angle and distance dependence of the superexchange.

In a first step the simulation cell in Fig. 4 was relaxed and allowed to change in shape and volume. The thereby-obtained structure was used for further calculations. Determining the distance dependence of the AFM coupling strength, the bond
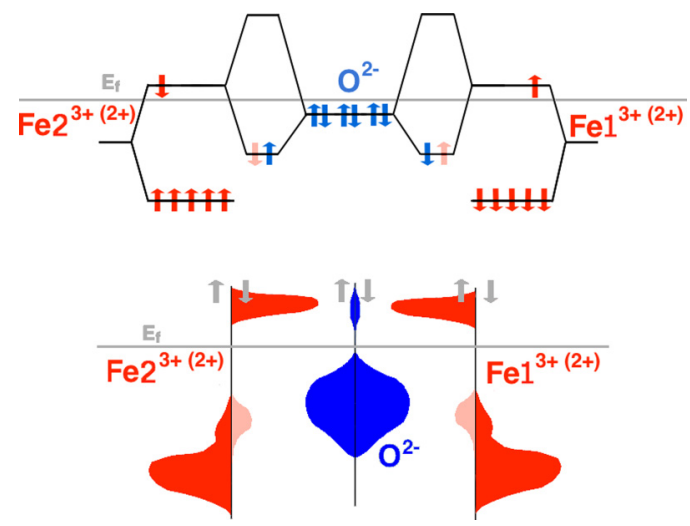

FIG. 3. (Color online) The upper part shows the MO diagram of an Fe1-O-Fe2 complex inside GFO. The lower part of the figure sketches the corresponding DOS. Marked in red and blue are the $\mathrm{Fe}$ $d$ and the $\mathrm{O} p$ states, respectively. Pale red denotes the additional $\mathrm{Fe}$ electron which becomes shifted below the Fermi energy by the Fe-O interaction. 

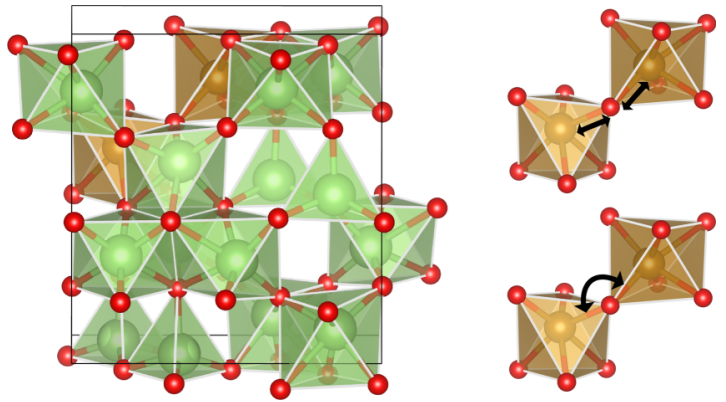

FIG. 4. (Color online) Unit cell with a single Fe1-O-Fe2 $\left(125.84^{\circ}\right)$ complex. Bond length and bond angle are varied to consider the alteration on the AFM superexchange.

length of the $\mathrm{Fe} 1-\mathrm{O}-\mathrm{Fe} 2$ complex was altered stepwise and kept fix during a second relaxation. The respective enclosed angle was kept constant at its equilibrium value obtained during the first relaxation. Investigating the angle dependence, the enclosed angle was varied but kept fixed during a second relaxation. The corresponding $\mathrm{Fe} 1-\mathrm{O}-\mathrm{Fe} 2$ distance was taken from the first relaxation and kept fixed as well.

These calculations were performed using the $\mathrm{GGA}+\mathrm{U}$ functional as described in the previous section.

The equilibirum bond length of the $\mathrm{Fe} 1-\mathrm{O}-\mathrm{Fe} 2$ structure is

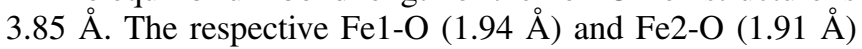
distances differ only slightly. The corresponding equilibrium angle is $125.84^{\circ}$.

Varying the bond length the $\mathrm{Fe} 1-\mathrm{O}$ and $\mathrm{Fe} 2-\mathrm{O}$ distances were stretched and compressed stepwise up to $20.0 \%$. This leads to a maximum and minimum bond length of about $2.3 \AA$ and $1.5 \AA$ for the particular $\mathrm{Fe}-\mathrm{O}$ bond. The equilibrium angle and the respective altered $\mathrm{Fe} 1-\mathrm{O}-\mathrm{Fe} 2$ distance were kept fix during a second relaxation of the cell. Figure 5(a) shows the AFM coupling strength designated as $E_{\text {diff }}[\mathrm{AFM}-\mathrm{FM}][\mathrm{eV} /$ cell $]$ as a function of the varied Fe1-O bond. The upper $x$ axis gives the total energy difference between the equilibrium ground state and the strained state.

According to the calculations performed the AFM coupling strength increases with decreasing bond length. A decrease in bond length leads to an enhancement of the Fe1-O-Fe2 orbital overlap and therefore to an augmented exchange

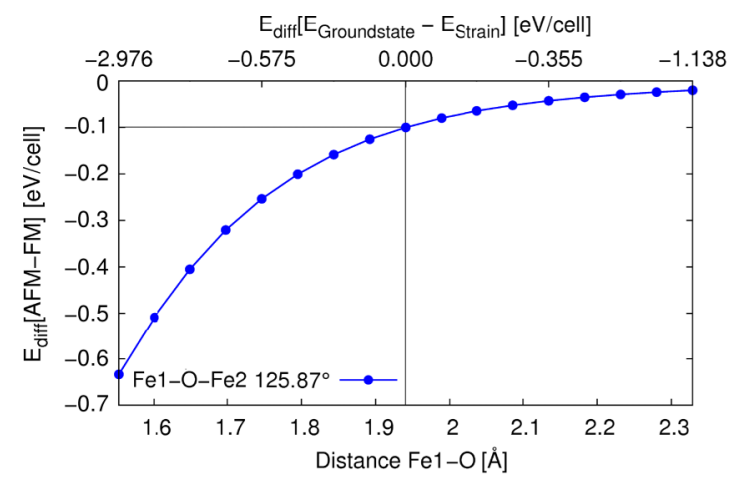

(a) between the atoms resulting in stronger AFM coupling. Correspondingly, an increase of the Fe-O distance causes the AFM superexchange to decrease.

The equilibrium bond angle of $125.84^{\circ}$ for the $\mathrm{Fe} 1-\mathrm{O}-\mathrm{Fe} 2$ bond was altered stepwise between $128.11^{\circ}$ and $122.23^{\circ}$ corresponding to $+1.8 \%$ and $-2.9 \%$, respectively. To calculate the energy as a function of the bond angle, the bond angle was kept fixed during a second relaxation while the other atoms of the cell and the shape and the volume of the simulation cell were allowed to relax. Figure 5(b) shows that with increasing angle the AFM coupling strength increases. Structural alterations bigger than the presented ones caused by angle and distance changes are not meaningful in terms of the phase stability.

However, Figs. 5(a) and 5(b) show that altering the enclosed angle does not affect the AFM coupling strength as much as changing the $\mathrm{Fe} 1-\mathrm{O}-\mathrm{Fe} 2$ bond length. Changing the enclosed angle causes a larger distortion of the oxygen octahedra than just varying the bond length. Increasing the bond angle rather than reducing it much earlier drives the system into a phase instability again because a larger bond angle leads to comparably higher distortions of the oxygen octahedra.

The obtained results are in good agreement with the GK rules which would predict that the AFM superexchange reduces with increasing bond length and decreasing angle due to a decrease of the orbital overlap between the interacting atoms.

\section{AFM superexchange in GFO: An additive quantity}

The total AFM coupling strength of pure GFO turns out to be closely equal to the sum of the AFM coupling strengths of the individual Fe1-O-Fe2 complexes inside the simulation cell. These complexes differ in enclosed angle and bond length. A representative example is given in Fig. 6.

Table II shows the corresponding AFM coupling strengths according to the respective angles shown in Fig. 6. The AFM coupling is the strongest for the (a) and (b) cases and almost by one half smaller for the (c) and (d) configurations. Even though we found out that increasing the enclosed angle and reducing the $\mathrm{Fe} 1-\mathrm{O}-\mathrm{Fe} 2$ bond length increases in the AFM coupling strength the (c) and (d) complexes show a reduced AFM superexchange.

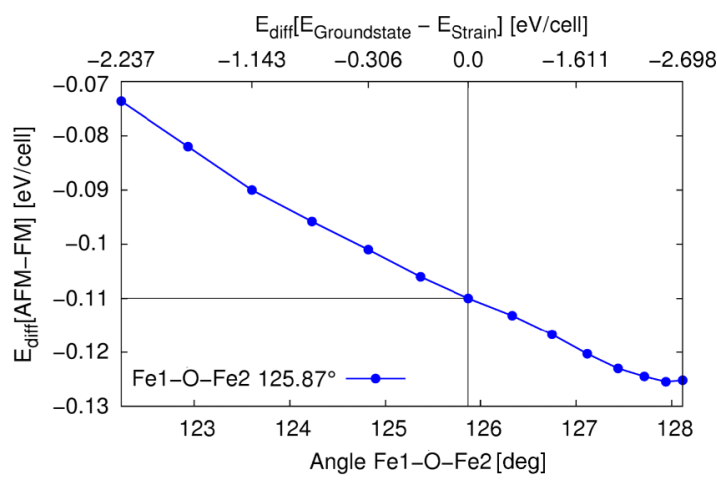

(b)

FIG. 5. (Color online) Plots (a) and (b) show the distance and the angle dependency of the AFM coupling strength, respectively. Increasing the distance and decreasing the angle leads to a decrease in AFM coupling strength. All energies are given per 40-atom simulation cell. 


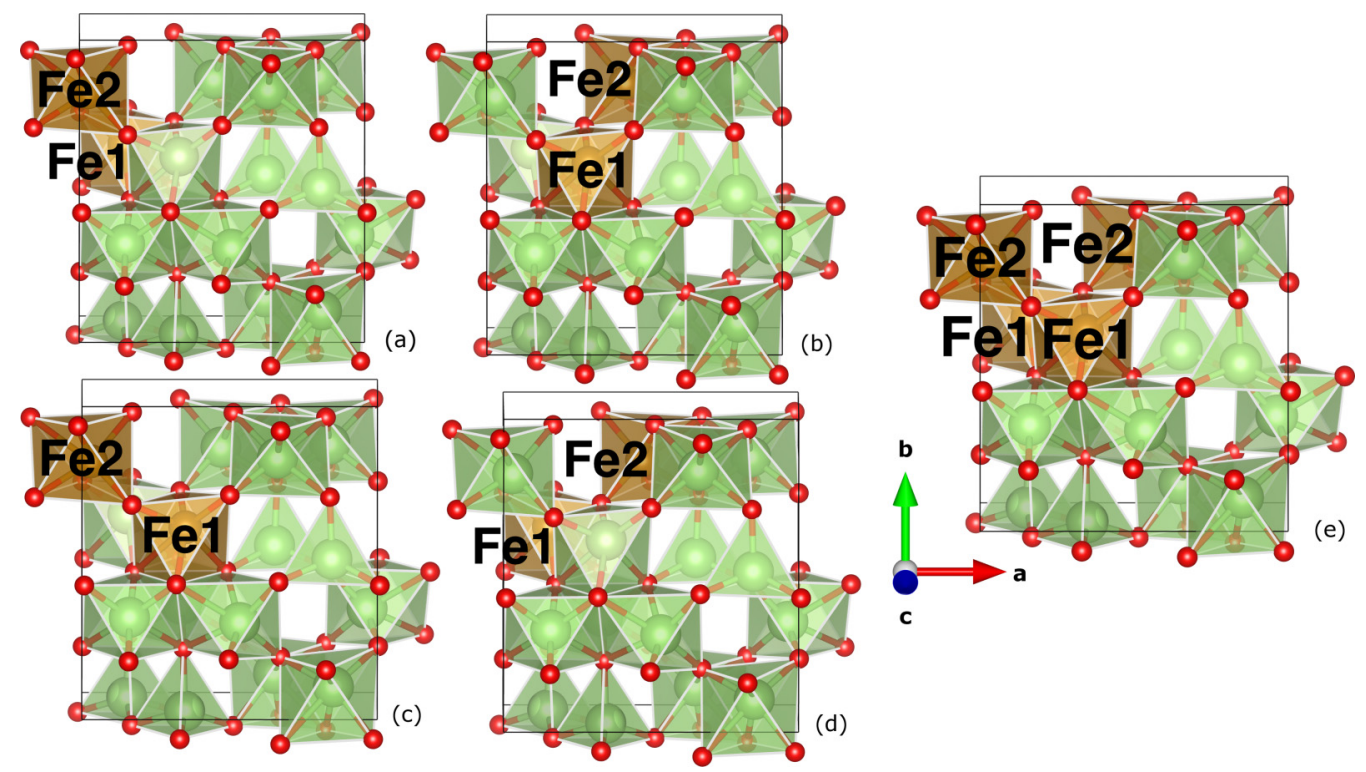

FIG. 6. (Color online) [(a)-(d)] Simulation cells with different single Fe1-O-Fe2 complexes. These complexes differ in their enclosed angle and bond length. The respective enclosed Fe1-O-Fe2 angles are $122.17^{\circ}$ for (a) and (b) and $125.84^{\circ}$ for (c) and (d). Case (e) is a modification which contains all complexes (a)-(d) in a single simulation cell. The total AFM coupling strength for configuration (e) turns out to the be close to the sum of the individual coupling strengths.

Given the small differences both in angle and bond length, this feature cannot be entirely related to the dependencies discussed in Fig. 6. The Fe atoms are surrounded by distorted oxygen octahedra that are tilted, stretched, and compressed in different directions. This of course effects the $\mathrm{Fe}-\mathrm{O}-\mathrm{Fe}$ orbital overlap and leads to the calculated behavior. In the (c) and (d) complexes the orbital overlap is reduced as compared to the (a) and (b) configurations where the coupling is stronger.

Case (e) includes all Fe1-O-Fe2 complexes shown in (a)-(d). Their AFM coupling strength is almost the sum of the single configurations, indicating that the AFM superexchange appears to be an additive quantity.

GFO in total has four Fe1-O-Fe2 complexes that differ in angle and distance and contribute to the AFM superexchange. The two Fe1-O-Fe2 complexes with the two strongest AFM coupling strengths are given in Table II. In addition, there is an $\mathrm{Fe} 1-\mathrm{O}-\mathrm{Fe} 2$ complex with an enclosed angle of $166.16^{\circ}$ and a bond length of $4.68 \AA$ having an AFM coupling strength of $-0.035 \mathrm{eV}$ and a fourth weakly coupled complex with an angle of $102.52^{\circ}$, a bond length of $4.19 \AA$, and an AFM coupling strength of only $-0.008 \mathrm{eV}$. Both contributions to the AFM superexchange are small and almost negligible.

\section{The instrinsic AFM chain in GFO}

Concluding the obtained results so far there are two $\mathrm{Fe} 1-\mathrm{O}-\mathrm{Fe} 2$ complexes dominating the AFM superexchange inside GFO. These are the Fe1-O-Fe2 complexes with enclosed angles of $122.17^{\circ}$ and $125.84^{\circ}$ (see Table II). Geometrically, these two complexes are connected to each other forming an Fe-O-Fe twisted chain as shown in Fig. 7. Stoichiometric GFO contains two of these Fe-O-Fe chains as can be seen in Fig. 1 formed by the upper-left and lower-right Fe structures. The connection between these chains is mediated by the above-mentioned very weak $\mathrm{Fe} 1-\mathrm{O}-\mathrm{Fe} 2$ complexes. Figure 7 shows a particular Fe-O-Fe chain as it meanders through the crystal.

\section{E. Magnetization density in GFO}

Figure 8(a) shows the magnetization density (spin up minus spin down) of an Fe1-O-Fe2 complex with the highest AFM coupling strength and an enclosed angle of $122.17^{\circ}$. Red tones represent positive and blue ones indicate negative vaules of the magnetization. Accordingly, Fig. 8(a) shows a negative magnetization density around $\mathrm{Fe} 1$ and a positive density

TABLE II. AFM coupling strengths of the Fe1-O-Fe2 complexes drawn in Fig. 6. Interestingly, (e) equals the sum of the values in (a) to (d). The respective geometries are GGA+U relaxed.

\begin{tabular}{lccc}
\hline \hline & Enclosed angle $\left(^{\circ}\right)$ & Bond length $(\AA)$ & $E_{\text {diff }}[\mathrm{AFM}-\mathrm{FM}](\mathrm{eV})$ \\
\hline Fe1-O-Fe2 (a) & 122.17 & 3.86 & -0.210 \\
Fe1-O-Fe2 (b) & 122.17 & 3.86 & -0.210 \\
Fe1-O-Fe2 (c) & 125.84 & 3.85 & -0.113 \\
Fe1-O-Fe2 (d) & 125.84 & 3.85 & -0.113 \\
Fe1-O-Fe2 (e) & (a)-(d) & (a)-(d) & -0.648 \\
\hline \hline
\end{tabular}




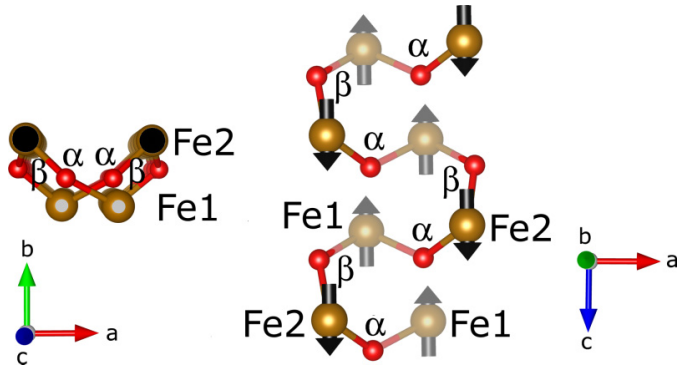

FIG. 7. (Color online) Dominant Fe1-O-Fe2 chain along the $c$ axis in GFO.

around the $\mathrm{Fe} 2$ atom. The $\mathrm{O}$ atom in between has a spin polarized $p$ orbital that is characterized by its dumbbell shape.

This dumbbell shape is induced by the covalent bond between the $\mathrm{O}$ atom and its two neighboring $\mathrm{Fe}$ atoms. The negative magnetization density between the $\mathrm{Fe} 2$ atom and the positive polarized $\mathrm{O} p$ orbital corresponds to the additional Fe $d$ electron that is shifted below the fermi energy by the superexchange interaction. This particular Fe $d$ electron is shown in Fig. 3 highlighted in pale red. It forms a molecular orbital with an $\mathrm{O} p$ electron. This $p$ electron shows reversed spin according to Hund's rules and is represented by the positive magnetization density (red) next to it. The AFM superexchange is mediated by this molecular orbital. Due to the spin compensation between the Fe electron and the $\mathrm{O}$ electron the spin density is rather small; however, an analysis of the energy-dependent electron density shows that this feature represents the $\mathrm{Fe}-\mathrm{O}$ states between $-5 \mathrm{eV}$ and the Fermi energy (see Fig. 2). The same is true for the positive magnetization density left-hand side of the Fe1 atom. It belongs to the additional $\mathrm{Fe} d$ electron that reduces the initial magnetic moment of $-5 \mu_{B}$ to about $-4 \mu_{B}$ per $\mathrm{Fe}$ atom. The negative magnetization density next to it again belongs to the $\mathrm{O} p$ electron with which the molecular orbital is formed with [Fig. 8(a)].

The plotted magnetization density in Fig. 8(b) shows two $\mathrm{O} p$ orbitals which differ in shape. The left-hand side $\mathrm{O}$ is part of the Fe1-O-Fe2 $\left(122.17^{\circ}\right)$ complex with the strongest AFM superexchange and the right-hand side $\mathrm{O}$ is part of the weakest $\mathrm{Fe} 1-\mathrm{O}-\mathrm{Fe} 2$ configuration with an enclosed angle of $102.52^{\circ}$. Since the $\mathrm{O}$ atoms mediate the superexchange, the shape of the O $p$ orbitals immediately indicates the strength of the AFM coupling. The shape of the left-hand side $\mathrm{O} p$ orbital forms a

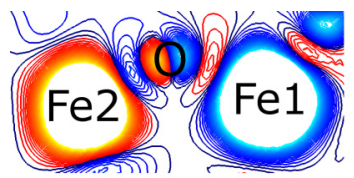

(a)

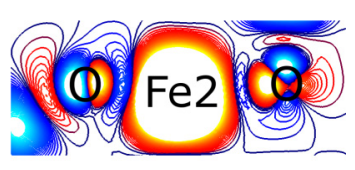

(b)
FIG. 8. (Color online) Panel (a) shows the magnetization density (spin up minus spin down) of an Fe1-O-Fe2 couple inside the GFO simulation cell. Denoted in red and blue tones are the positive and negative magnetization values, respectively. Panel (b) shows the magnetization density of an $\mathrm{Fe} 2$ atom being part of two $\mathrm{Fe} 1-\mathrm{O}-\mathrm{Fe} 2$ complexes. The left $\mathrm{O}$ atom is part of a strong AFM coupling strength $\mathrm{Fe} 1-\mathrm{O}-\mathrm{Fe} 2\left(122.17^{\circ}\right)$ complex and the right $\mathrm{O}$ atom of a weak Fe1-O-Fe2 $\left(102.52^{\circ}\right)$ configuration. straight $\sigma$ bond between its neighboring $\mathrm{Fe} 1$ and $\mathrm{Fe} 2$ atom. The shape of the right-hand side $\mathrm{O} p$ orbital mediating almost no AFM coupling resembles a $90^{\circ}$ bonding. According to the GK rules a bond along a $90^{\circ}$ angle should couple ferromagnetically. Since in GFO we observe superexchange along an Fe1-O-Fe2 chain the AFM order is retained; however, the AFM coupling strength along the $102.52^{\circ}$ angle becomes only reduced.

\section{F. Magnetocrystalline anisotropy energy in GFO}

MAE is another interesting property of GFO. According to experiment the $c$ axis of pure GFO is the easy axis and corresponds to the ground state direction of the magnetic moments while the hard axis is in $b$ direction [1,14]. F. Ibrahim and M. Alounani [15] already discussed the impact of excess $F e$ and the effect of varying $U$ values in the GGA+U approximation on the calculated MAE. Independent of the $U$ value the MAE decreases with increasing amount of excess $\mathrm{Fe}$ atoms. We focus on the partly predicted and experimentally measured $[6,14]$ but, to the best of our knowledge, not yet theoretically studied sublattice-dependent MAE.

Calculating the MAE of the $a, b$, and $c$ axes in pure GFO the simulation cells were both relaxed using the HSE functional and the GGA+U approximation. Hence, we could investigate the effect of the used exchange and correlation functional on the resulting crystal structure and the MAE.

The first entry in Table III shows the energy difference of the [001] and [100] configurations. In the [001] configuration the magnetic moments of both the Fe1 and the Fe2 sublattices are oriented along the ground-state $c$ direction. This energy is then subtracted from the energy of the [100] configuration where the magnetic moments of both sublattices are rotated in the $a$ direction. The second row in Table III gives the energy difference of the [001] and [010] configurations. Here the magnetic moments are rotated first in the $c$ direction and then in the $b$ direction. The calculated MAE of pure GFO is in good agreement with experiment and previous calculations $[14,15]$. Both the HSE and GGA $+\mathrm{U}$ relaxed crystal structures define the $c$ axis as the easy axis and the $b$ axis as the hard axis. The HSE-relaxed geometry shows a larger MAE for the $b$ axis, making it less favorable. In the "Ga2-Fe1" simulation cell a Ga2 atoms exchanges its site with an Fe1 atom. The corresponding simulation cell has a net magnetic moment unequal zero, leading to a ferrimagnetic ground state of $5 \mu \mathrm{B}$ per simulation cell. A more detailed discussion of the different inner cationic site disorder configurations and their respective energies are given in Sec. IV. Interestingly this configuration leads to a change of the easy axis of the system, which is now the $a$ axis. This is true for both the HSE and the GGA+U relaxed geometries, while HSE again yields larger anisotropies. Focusing on a further clarification of a sublattice-dependent anisotropy, the magnetic moments of the $\mathrm{Fe} 1$ and $\mathrm{Fe} 2$ sublattice atoms were rotated against each other and the respective configuration energy was calculated. First, a calculation was performed where the magnetic moments of the Fel sublattice atoms are rotated in the $a$ direction while the moments of the $\mathrm{Fe} 2$ atoms are fixed in the $c$ direction, followed by another calculation where the magnetic moments of the $\mathrm{Fe} 2$ sublattice atoms are rotated in the $a$ direction and the magnetic moments of the Fe1 atoms are held in the $c$ direction. 
TABLE III. MAE of pristine and inner cationic site-disorder-affected GFO.

\begin{tabular}{lcccc}
\hline \hline MAE (meV) & GFO (HSE) & GFO (GGA+U) & Ga2-Fe1 (HSE) & Ga2-Fe1 (GGA+U) \\
\hline$[001]-[100]$ & -0.230 & -0.182 & -0.434 & -0.423 \\
{$[001]-[010]$} & -0.291 & -0.212 & -0.357 & -0.309 \\
Fe1[100]Fe2[001] - Fe1[001]Fe2[100] & -0.153 & -0.163 & -1.765 & -1.655 \\
Fe1[010]Fe2[001]- Fe1[001]Fe2[010] & -0.012 & -0.028 & -1.668 & -1.522 \\
Fe1[100]Fe2[001]- Fe1[010]Fe2[001] & -0.088 & -0.060 & -0.046 & -0.044 \\
Fe1[001]Fe2[100]- Fe1[001]Fe2[010] & 0.053 & 0.076 & 0.051 & 0.090 \\
\hline \hline
\end{tabular}

Computing the energy difference of these two configurations makes it possible to determine a potential sublattice-dependent magnetic anisotropy. The same was done rotating the magnetic moments of the sublattices vice versa in the $b$ direction, keeping the respective other one fixed in the ground-state $c$ direction. In both pure GFO and in the case of inner cationic site disorder the Fe1 sublattice is less anisotropic than the Fe2 sublattice. This was also predicted in experiment by Reddy et al. [6]. The anisotropy between the $\mathrm{Fe} 1$ and $\mathrm{Fe} 2$ sublattices is more pronounced in the $a$ direction. However, the overall sublattice-dependent anisotropy is one order of magnitude smaller in pure GFO. It was also investigated whether the magnetic moments of the $\mathrm{Fe} 1$ and $\mathrm{Fe} 2$ sublattices prefer being rotated in the $a$ direction or in the $b$ direction. Starting with the $\mathrm{Fe} 1$ sublattice the magnetic moments were once turned in the $a$ direction and then in the $b$ direction, keeping the magnetic moments of the $\mathrm{Fe} 2$ sublattice fixed in the ground-state $c$ direction. It shows that the Fe1 sublattice moments prefer being rotated in the $a$ direction over the $b$ direction. The same was done with the Fe 2 sublattice magnetic moments. In that case, the magnetic moments prefer being rotated in the $b$ direction rather than in the $a$ direction.

\section{CATION DOPING: RANGING FROM $\varepsilon$-GO TO GFO AND $\varepsilon$-FO}

\section{A. From $\varepsilon$-GO to GFO: $0.0 \leqslant x \leqslant 1.0$}

The magnetic properties of $\mathrm{Ga}_{2-x} \mathrm{Fe}_{x} \mathrm{O}_{3}$ were investigated for a doping concentration of $0.0 \leqslant x \leqslant 2.0$ [36].

At a concentration of $x=0.0 \mathrm{GFO}$ turns into nonmagnetic isomorphic $\varepsilon$-GO. Raising the doping concentration stepwise up to $x=1.0$ by adding $\mathrm{Fe}$ atoms to the $\varepsilon$-GO cell gives stoichiometric GFO. Reaching the value of $x=2.0$, where all $\mathrm{Ga}$ atoms are exchanged by $\mathrm{Fe}$ atoms, turns the GFO simulation cell into the AFM isomorphic $\varepsilon$-FO structure (Fig. 9). The respective calculated lattice parameters and band

$\varepsilon-\mathrm{GO}$
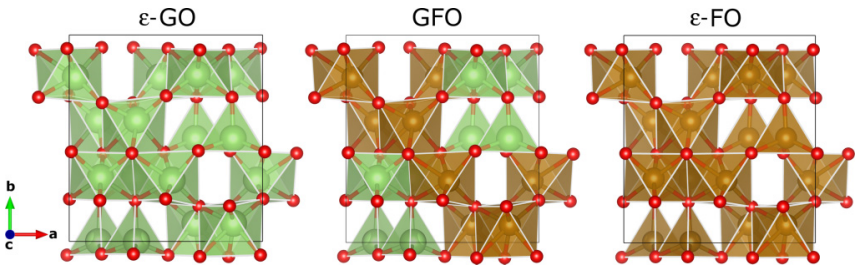

FIG. 9. (Color online) Shown are the respective simulation cells of $\mathrm{Ga}_{2-x} \mathrm{Fe}_{x} \mathrm{O}_{3}$ for a doping concentration of $x=0.0, x=1.0$, and $x=2.0$. These are equivalent to the crystal structures of $\varepsilon$-GO, GFO, and $\varepsilon$-FO. gaps are given in Table I. In addition, we also studied the effect of inner cationic site disorder on the magnetic properties and the phase stability of stoichiometric GFO.

At a cation-doping concentration of $x=0.1$ one $\mathrm{Fe}$ is added to the $\varepsilon$-GO simulation cell. There exist four different lattice sites on which the Fe atom can be placed. To denote the respective doping site of the introduced $\mathrm{Fe}$ atom we use the Fe1/2 and Ga1/2 site notation of stoichiometric GFO (Fig. 1). The additional $\mathrm{Fe}$ atom prefers an $\mathrm{Fe} 1$ site over an $\mathrm{Fe} 2$ site by about $31 \mathrm{meV}$. Placing the $\mathrm{Fe}$ atom on a $\mathrm{Ga} 1$ or $\mathrm{Ga} 2$ site is by 359 and $112 \mathrm{meV}$ less favorable as compared to the occupation of an Fe1 site.

Adding a second Fe atom $(x=0.2)$ to the $\varepsilon$-GO cell offers a multitude of doping configurations. In addition to the already-discussed Fe1-O-Fe2 configurations, we investigated the magnetic properties of possible $\mathrm{Fe} 1-\mathrm{O}-\mathrm{Fe} 1, \mathrm{Fe} 2-$ $\mathrm{O}-\mathrm{Fe} 2$, and $\mathrm{Fe}-\mathrm{O}-\mathrm{Fe}(\mathrm{Ga} 1 / 2)$ configurations, where the latter means that an $\mathrm{Fe}$ sits either on a $\mathrm{Ga} 1$ or a $\mathrm{Ga} 2$ position. The calculated AFM coupling strengths of the Fe1-O-Fe1 and Fe2-O-Fe2 complexes are very small with 0.062 and $0.004 \mathrm{meV}$, respectively. Comparing their phase stabilities to the most stable $\mathrm{Fe} 1-\mathrm{O}-\mathrm{Fe} 2\left(122.17^{\circ}\right)$ configuration (see Table II) shows that the formation of an Fe1-O-Fe1 complex is less favorable only by $17 \mathrm{meV}$. The Fe2-O-Fe 2 configuration has a higher energy difference of about $139 \mathrm{meV}$. The most stable $\mathrm{Fe}-\mathrm{O}-\mathrm{Fe}(\mathrm{Ga} 1 / 2)$ configurations are the $\mathrm{Fe} 1-\mathrm{O}-\mathrm{Fe}(\mathrm{Ga} 2)$ and the $\mathrm{Fe} 2-\mathrm{O}-\mathrm{Fe}(\mathrm{Ga} 1)$ configuration, with an enclosed angle of $163.56^{\circ}$ and $122.53^{\circ}$, respectively. The first configuration is $250 \mathrm{meV}$ more stable than the latter one, whereas the latter has a 55-meV-larger AFM coupling strength. The most stable Fe1-O-Fe2 configuration is still more favorable than the $\mathrm{Fe} 1-\mathrm{O}-\mathrm{Fe}(\mathrm{Ga} 2)$ configuration by $153 \mathrm{meV}$. Regarding the calculated phase stabilities of the investigated doping configurations, two $\mathrm{Fe}$ atoms would first form an $\mathrm{Fe} 1-\mathrm{O}-\mathrm{Fe} 2$ configuration. This is followed by an $\mathrm{Fe} 1-\mathrm{O}-\mathrm{Fe} 1$ configuration, an $\mathrm{Fe} 2-\mathrm{O}-\mathrm{Fe} 2$ configuration and an $\mathrm{Fe} 1-\mathrm{O}-\mathrm{Fe}(\mathrm{Ga} 2)$ configuration. Occupying two $\mathrm{Ga}$ sites forming a $\mathrm{Fe}(\mathrm{Ga} 1 / 2)-\mathrm{O}-$ $\mathrm{Fe}(\mathrm{Ga} 1 / 2)$ complex is at least $250 \mathrm{meV}$ less probable than the energetically best Fe1-O-Fe2 configuration. No ferromagnetic ground state can be produced with any doping configurations at $x=0.2$.

At $x=0.4$ it is most favorable in energy when two $\mathrm{Fe}$ atoms occupy two adjacent $\mathrm{Fe} 1$ sites and the third $\mathrm{Fe}$ atom occupies a next-nearest $\mathrm{Fe} 2$ site. The $\mathrm{Fe} 1$ atoms have a negative magnetic moment of about $-4 \mu_{B}$ each and the $\mathrm{Fe} 2$ atom a positive magnetic moment of about $+4 \mu_{B}$, as expected from the arrangement of the magnetic moments in stoichiometric GFO. This leads to a total magnetic moment of $-5 \mu_{B}$ per simulation cell and a ferrimagnetic ground state. It should 


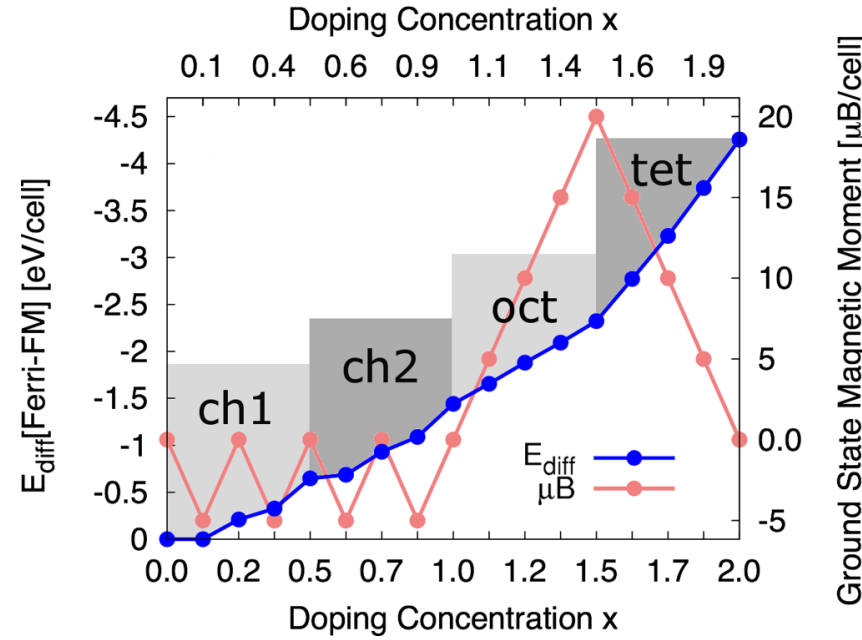

FIG. 10. (Color online) We show the AFM coupling strength $E_{\text {diff }}$ (blue) and the corresponding ground state magnetic moment (red) as a function of the doping concentration $x$. In the range of $x=0.0$ to $x=0.5$ the first Fe1-O-Fe2 chain in formed in GFO denoted by "ch1" (chain 1). In the "ch2" highlighted area the second Fe1-O-Fe2 chain is formed, where $E_{\text {diff }}$ for "ch1" and the "ch2" shows the same behavior. In the area "oct" (octahedral) all Ga2 sites are substituted by $\mathrm{Fe}$ atoms. Here the slope is constant, indicating the additive character of the AFM coupling strength of the formed $\mathrm{Fe} 1-\mathrm{O}-\mathrm{Fe}(\mathrm{Ga} 2)$ configurations. In the area "tet" (tetrahedral) all Ga1 sites become stepwise occupied by Fe atoms. As before we find an almost-linear increase in the coupling strength.

be noted that this total magnetic moment also contains the contributions from the oxygens.

Four substitutional Fe atoms are added to the $\varepsilon$-GO at a doping concentration of $x=0.5$. In terms of phase stability they are best arranged in an Fe1 and Fe2 cluster [Fig. 6(e)], forming the earlier discussed Fe1-O-Fe2 chain (Fig. 7).

Adding stepwise another four $\mathrm{Fe}$ atoms to a doping concentration of $x=1.0$, a second $\mathrm{Fe} 1-\mathrm{O}-\mathrm{Fe} 2$ chain is formed following the same mechanism as before.

For a doping concentration of $x=0.0$ to $x=1.0$ the total magnetic moment per simulation cell thus turns out to be either $0 \mu_{B}$ or $-5 \mu_{B}$ (Fig. 10).

\section{B. Inner cationic site disorder in stoichiometric GFO}

Experimental studies also report to find a ferrimagnetic state in GFO beside the expected AFM ground state of stoichiometric GFO. These findings can be attributed to the presence of an inner cationic site disorder where $\mathrm{Ga}$ and $\mathrm{Fe}$ atoms exchange cell sites. Recently performed calculations also are reported to be in better agreement with experiment if they include an inner cationic site disorder in their systems $[7,37,38]$. We investigate the possible inner cationic site disorder configurations in stoichiometric GFO and calculate their phase stabilities and total magnetic moment.

The energetically most favorable site disorder is the Fe2$\mathrm{Ga} 2$ exchange where an $\mathrm{Fe} 2$ atom exchanges site with a $\mathrm{Ga} 2$ atom. Compared to stoichiometric GFO showing no inner cationic site disorder the loss of phase stability is about $157 \mathrm{meV}$. The AFM coupling strength reduces from 1.443 to
$1.302 \mathrm{eV}$ per simulation cell and has a total magnetic moment of $0 \mu_{B}$. In that particular case no ferrimagnetic ground state is introduced.

The case of an Fe1-Ga2 exchange leads to a loss in energy compared to pristine GFO of $183 \mathrm{meV}$ and indeed yields a ferrimagnetic ground state with $5 \mu_{B}$ per simulation cell.

Followed by that are the Fe2-Ga1 and Fe1-Ga1 exchange configurations. Both differ only by $10 \mathrm{meV}$ in phase stability but are about $400 \mathrm{meV}$ less favorable than pristine GFO. The increased loss in phase stability is due to the different $\mathrm{O}$ environment of $\mathrm{Ga} 1$ since Fe prefers being surrounded by an $\mathrm{O}$ octahedron rather than by an $\mathrm{O}$ tetrahedron. However, the Fe2-Ga1 exchange again leads to a ferrimagnetic ground state of $-5 \mu_{B}$ per simulation cell.

The magnetic moment of an Fe atom occupying a Ga1 or $\mathrm{Ga} 2$ site equals the one at an $\mathrm{Fe} 1$ or $\mathrm{Fe} 2$ site, respectively. The Fe1-Ga2 exchange is the energetically most favorable inner cationic site disorder configuration, leading to a ferrimagnetic ground state. This is the reason why its effect on the MAE is calculated and discussed in Sec. III.

\section{From GFO to $\varepsilon$-FO: $1.0 \leqslant x \leqslant 2.0$}

As discussed previously, the Fe atoms prefer the occupation of $\mathrm{Ga} 2$ over $\mathrm{Ga} 1$ sites. Hence, ranging from a doping concentration of $x=1.0$ to $x=1.5$, all Ga2 sites are occupied first.

Substituting a Ga2 site forms an additional Fe1-O-Fe(Ga2) bond inside GFO which is about $232 \mathrm{meV}$ energetically more stable than the formation of an $\mathrm{Fe} 2-\mathrm{O}-\mathrm{Fe}(\mathrm{Ga} 1)$ complex where a Ga1 site is occupied by an excess Fe atom. However, the latter is about $57 \mathrm{meV}$ stronger in AFM coupling strength.

Increasing the doping concentration further up to a value of $x=2.0$ all Ga1 are occupied until no $\mathrm{Ga}$ atom is left inside the simulation cell. GFO turns into the $\varepsilon$-FO structure.

Figure 10 shows the AFM coupling strength as a function of the doping concentration $x$ and the corresponding total magnetic moment of the respective simulation cell. For each concentration $x$ (Fig. 10) the values of the lowest energy for the possible doping configurations are plotted.

The additive character of the AFM coupling strength can be nicely seen in Fig. 10. Taking a closer look at the slope for a doping concentration of $0.1 \leqslant x \leqslant 1.0$, we see the same behavior of the curve in the range of $0.1 \leqslant x \leqslant 0.5$ and $0.6 \leqslant$ $x \leqslant 1.0$. Here the two Fe1-O-Fe 2 chains are formed inside the simulation cell. The first one in a range of $0.1 \leqslant x \leqslant 0.5$ and the second one for $0.6 \leqslant x \leqslant 1.0$. The magnetic moment per simulation cell alternates between $0 \mu_{B}$ or $-5 \mu_{B}$.

For a doping concentration of $1.1 \leqslant x \leqslant 1.5$ the $\mathrm{Ga} 2$ becomes substituted and additional $\mathrm{Fe} 1-\mathrm{O}-\mathrm{Fe}(\mathrm{Ga} 2)$ configurations are formed. The slope of the curve shows an almost linear increase of the AFM coupling strength and again indicates the additive character of this property. The same is true for the range of $1.6 \leqslant x \leqslant 2.0$ where all Ga1 sites are substituted and $\mathrm{Fe} 1-\mathrm{O}-\mathrm{Fe}(\mathrm{Ga} 1)$ complexes are formed.

The total magnetic moment is maximal at $x=1.5$ and yields $20 \mu_{B}$ per simulation cell. At $x=2.0$ when transforming into the $\varepsilon$-FO structure we end up in an AFM ground state.

The defect formation energy provides information on the phase stability of the doped system. According to the following 


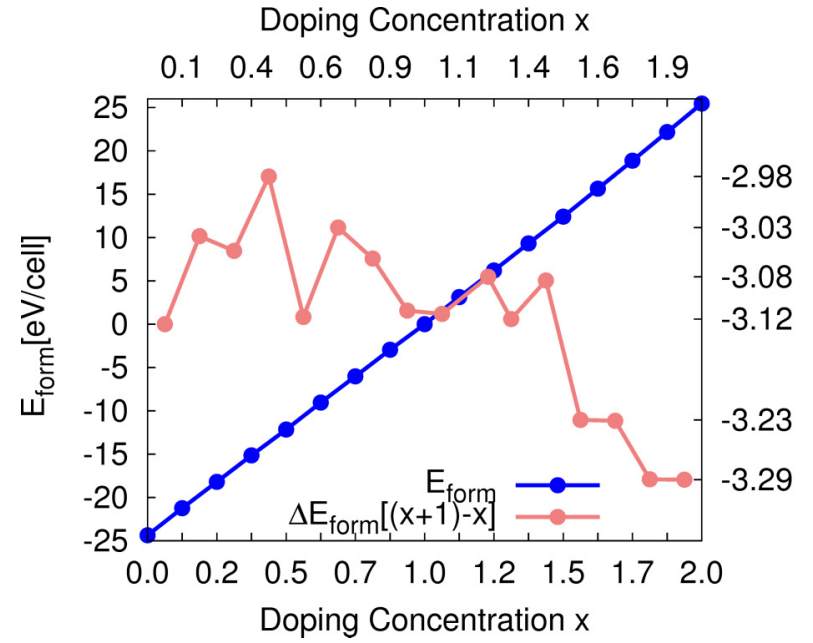

FIG. 11. (Color online) We show the defect formation energy $E^{\text {form }}$ (blue) as a function of the doping concentration $x$ and the difference $\Delta E_{\text {form }}$ (red) (see text).

formula [39]:

$$
E^{\text {form }}[x]=E_{\mathrm{tot}}[x]-E_{\mathrm{tot}}[\text { bulk }]+n\left(\mu_{\mathrm{Ga}}-\mu_{\mathrm{Fe}}\right),
$$

where $E_{\text {tot }}$ is the total free energy of the cation-doped simulation cell. $x$ is the doping concentration and $E_{\text {tot }}[\mathrm{bulk}]$ stands for the total free energy of the pristine GFO simulation cell. $n$ indicates the number of $\mathrm{Ga}$ atoms that are replaced by $\mathrm{Fe}$ atoms. $\mu_{\mathrm{Ga}}$ and $\mu_{\mathrm{Fe}}$ are the corresponding chemical potentials. The reference energies of the chemical potentials are calculated from the $\alpha$-Fe and $\alpha$-Ga phases.

Figure 11 shows the defect formation energy of the cation doping referenced to the energy of the pristine GFO simulation cell.

The defect formation energy shows almost linear behavior. A detailed account of the deviations of the linearity is shown by the red curve in Fig. 11 (note the much smaller energy scale). It shows the energy difference of two consecutive configurations which differ by one Fe atom, $\Delta E_{\text {form }}=E_{\text {form }}[x+$ $1]-E_{\text {form }}[x]$. The average value for the replacement of one Fe for one $\mathrm{Ga}$ is $\Delta E_{\text {form }}=3.18 \mathrm{eV}$.

\section{THE EFFECT OF STRAIN}

Straining the simulation cell induces a distortion of the ground-state cell geometry. Intrinsic angles and atomic distances are altered and hence can lead to a change of the magnetic and optical properties; the latter ones were discussed by Roy et al. [37]. However, in the present investigation we are interested in the effect of strain on the AFM coupling strength in pristine GFO and include inner cationic site disorder and cation doping concentrations of $0.9 \leqslant x \leqslant 1.4$.

\section{A. Strain on pristine GFO}

Starting with pristine GFO, the simulation cell is stretched and compressed in the $a, b$, and $c$ directions, respectively. The volume of the cell is preserved. The simulated strain on the lattice parameters is in a range of +3.5 to $-3.5 \%$ with respect to the calculated ground-state cell parameters. Figure 12

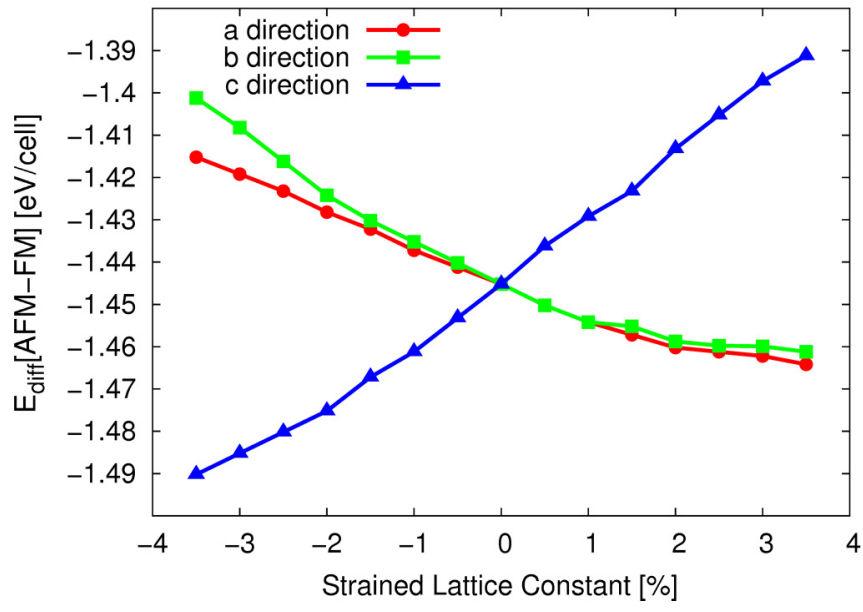

FIG. 12. (Color online) AFM coupling strength $E_{\text {diff }}$ as a function of the strained lattice constant in the $a$ (red), $b$ (green), and $c$ (blue) directions.

shows the respective change of the AFM coupling strength. Further information on the strain-induced geometry changes and distorted $\mathrm{Fe}-\mathrm{O}-\mathrm{Fe}$ bonds are given in the Supplemental Material SM2 [40].

Compressing the simulation cell in the $a$ direction or $b$ direction reduces the AFM coupling strength. Decreasing the length of the lattice $c$ parameter, on the other hand, increases the coupling. The reversed increase and decrease of the AFM coupling strength in the $c$ direction can be explained by the geometric distortions induced in the simulation cell.

While some of the Fe1-O-Fe2 angles and distances become smaller or bigger, stretching and compressing the simulation cell along the $a$ direction or $b$ direction, they oppositely become bigger or smaller, stretching the system in the $c$ direction.

Compressing the cell in the $a$ and $b$ directions up to $-3.5 \%$ leads to a decrease in the AFM coupling by 31 and $44 \mathrm{meV}$ per simulation cell, respectively. Stretching the cell up to $+3.5 \%$ enhances the coupling by $19 \mathrm{meV}$ for the $a$ direction and $16 \mathrm{meV}$ for the $b$ direction. The AFM coupling strength increases compressing the cell in the $c$ direction by $54 \mathrm{meV}$ and decreases with extension by $45 \mathrm{meV}$ per simulation cell.

Straining the simulation in cell in the $c$ direction leads to the biggest change in the AFM coupling strength.

The loss in total energy and phase stability of the strained simulation cell correlates with the absolute change of the AFM coupling strength. The higher the increase/decrease of the AFM coupling strength caused by the applied strain the bigger/smaller becomes the phase stability.

Compressing the cell in the $a$ direction reduces the total energy per simulation cell by $336 \mathrm{meV}$ compared to the unstrained configuration. Stretching leads to an energy loss of $292 \mathrm{meV}$ per simulation cell. In the $b$ direction the total energy of the strained simulation cell becomes decreased by 351 and $358 \mathrm{meV}$ for compressing and stretching the system, respectively. The biggest loss in phase stability is caused by stretching and compressing the cell in the $c$ direction. Stretching and compressing the cell decreases the cell energy by 382 and $332 \mathrm{meV}$ per simulation cell, respectively. 


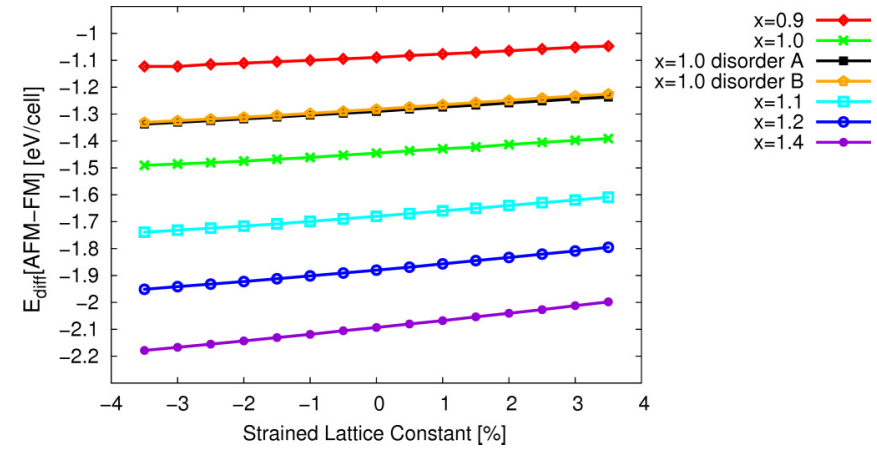

FIG. 13. (Color online) Straining the $\mathrm{Ga}_{2-x} \mathrm{Fe}_{x} \mathrm{O}_{3}$ simulation cell in the $c$ direction for a doping concentration of $x=0.9$ to $x=1.4$, including the case of inner cationic site disorder. Plotted is the AFM coupling strength $E_{\text {diff }}$ as a function of strain.

\section{B. Strain on cation-doped $\mathrm{Ga}_{2-x} \mathrm{Fe}_{x} \mathrm{O}_{3}$}

The effect of strain in cation-doped $\mathrm{Ga}_{2-x} \mathrm{Fe}_{x} \mathrm{O}_{3}$ was investigated for a doping concentration between $x=0.9$ and $x=1.4$. This corresponds to the cation doping range investigated in most experiments. Since strain along the $c$ axis has the biggest impact on the AFM coupling strength strain was applied in the $c$ direction in the following calculations. For each doping concentration the previously determined energetically most stable doping configuration is used. In addition, we investigate two cases of inner cationic site disorder. The most stable ones are the $\mathrm{Fe} 2-\mathrm{Ga} 2$ site exchange leading to a total magnetic moment of zero and the $\mathrm{Fe} 1-\mathrm{Ga} 2$ site exchange causing the GFO ground state to become ferrimagnetic with $+5 \mu_{B}$ per simulation cell.

Figure 13 shows the calculated AFM coupling strength as a function of the applied strain.

For all investigated configurations the AFM coupling strength increases upon compressing the corresponding simulation cell in the $c$ direction. The overall coupling strength increases with increasing Fe content. This can be seen by the downward shift to larger coupling strengths of the respective curves for higher cation doping concentrations $x$. The plotted curves have a steeper rise for higher Fe concentrations. The two investigated cases of inner cationic site disorder are almost equal in energy.

\section{CONCLUSION}

The performed calculations provide a detailed insight into the origin of the AFM superexchange mechanism in stoichiometric and cation-doped GFO. The MO diagram given in Fig. 3 shows that molecular orbitals are formed between the $\mathrm{O}$ and $\mathrm{Fe}$ atoms. The $\mathrm{Fe}-\mathrm{O}$ interaction reduces the formally $5 \mu_{B}$ for an $\mathrm{Fe} 3^{+}$state to the observed about $4 \mu_{B}$. The AFM superexchange is mediated by these covalent bonds.

Further, we show that the AFM superexchange follows, in principle, the GK rules. The AFM exchange enhances with increasing enclosed angle and decreasing bond length due to an enhanced orbital overlap.

The magnetization density given in Fig. 8(b) reflects this behavior. According to the GK rules we find stronger AFM coupling for bond angles closer to $180^{\circ}$ and a subsequent weakening when the bond angle approaches $90^{\circ}$.

The AFM superexchange throughout the crystal is carried forward by an $\mathrm{Fe} 1-\mathrm{O}-\mathrm{Fe} 2$ chain. This particular chain is built up by two $\mathrm{Fe} 1-\mathrm{O}-\mathrm{Fe} 2$ complexes which differ in their enclosed angle and bond length (Fig. 7).

MAE is another interesting property of GFO. The calculated MAE and the obtained magnetic easy and hard axis are in agreement with experiment. The magnetic easy and hard axis are found to be along the $c$ and $b$ directions, respectively. Relaxing the GFO simulation cell within the HSE approximation yields larger anisotropies. The presence of an inner cationic site disorder can lead to a ferrimagnetic ground state. In that case the magnetic hard axis changes to the $a$ direction.

Cation doping increases the AFM coupling strength. The magnetic moment per simulation cell is a maximum for a doping concentration of $x=1.5$. In this configuration all $\mathrm{Ga} 2$ sites are occupied by four excess $\mathrm{Fe}$ atoms leading to a magnetic moment of $20 \mu_{B}$ per simulation cell.

Figure 10 shows the additive character of the AFM coupling. Starting with the $\varepsilon$-GO structure the two Fe1-O$\mathrm{Fe} 2$ chains are formed first. Increasing the cation doping concentration the $\mathrm{Ga} 2$ sites are occupied followed by the $\mathrm{Ga} 1$ sites until the $\varepsilon$-FO phase is formed.

Strain distorts the cell geometry and alters the intrinsic bond lengths and angles which can affect the AFM coupling strength. The biggest effect on the AFM coupling strength is obtained by stretching and compressing the pristine GFO simulation cell in the $c$ direction. Figure 13 shows the effect of cation doping on the strained simulation cell. The slopes of the curves are shifted to higher coupling strengths with increasing doping concentration $x$. The respective defect formation energy is almost constant with a mean value of $3.18 \mathrm{eV}$.

\section{ACKNOWLEDGMENTS}

The authors acknowledge support from the Austrian Science Fund FWF within SFB ViCoM F4109-N13 P09.
[1] T. Arima, D. Higashiyama, Y. Kaneko, J. He, T. Goto, S. Miyasaka, T. Kimura, K. Oikawa, T. Kamiyama, R. Kumai, and Y. Tokura, Structural and magnetoelectric properties of $\mathrm{Ga}_{2-x} \mathrm{Fe}_{x} \mathrm{O}_{3}$ single crystals grown by a floating-zone method, Phys. Rev. B 70, 064426 (2004).

[2] Yu. F. Popov, A. M. Kadomtseva, G. P. Vorobev, V. A. Timofeeva, D. M. Ustinin, A. K. Zvezdin, and M. M. Tegeranchi, Magnetoelectric effect and toroidal ordering in $\mathrm{Ga}_{2-x} \mathrm{Fe}_{x} \mathrm{O}_{3}$, J. Exp. Theor. Phys. 87, 146 (1998).
[3] Somdutta Mukherjee, Amritendu Roy, Sushil Auluck, Rajendra Prasad, Rajeev Gupta, and Ashish Garg, Room Temperature Nanoscale Ferroelectricity in Magnetoelectric $\mathrm{GaFeO}_{3}$ Epitaxial Thin Films, Phys. Rev. Lett. 111, 087601 (2013).

[4] J. P. Remeika, $\mathrm{GaFeO}_{3}$ : A ferromagnetic-piezoelectric compound, J. Appl. Phys. 31, S263 (1960).

[5] Christophe Lefevre, Francois Roulland, Alexandre Thomasson, Christian Meny, Florence Porcher, Gilles André, and Nathalie 
Viart, Magnetic and polar properties' optimization in the magnetoelectric $\mathrm{Ga}_{2-x} \mathrm{Fe}_{x} \mathrm{O}_{3}$ compounds, J. Phys. Chem. C 117, 14832 (2013).

[6] V. Raghavendra Reddy, Kavita Sharma, Ajay Gupta, and A. Banerjee, Magnetic anisotropy and sub-lattice magnetization study of polycrystalline magneto-electric $\mathrm{Ga}_{2-x} \mathrm{Fe}_{x} \mathrm{O}_{3}$, J. Magn. Magn. Mater. 362, 97 (2014).

[7] Alexandre Thomasson, Fatima Ibrahim, Christophe Lefevre, Emmanuel Autissier, François Roulland, Christian Mény, Cédric Leuvrey, Sukgeun Choi, William Jo, Olivier Crégut, Gilles Versini, Sophie Barre, Mébarek Alouani, and Nathalie Viart, Effects of iron concentration and cationic site disorder on the optical properties of magnetoelectric gallium ferrite thin films, RSC Adv. 3, 3124 (2013).

[8] E. Tronc, C. Chanéac, and J. P. Jolivet, Structural and magnetic characterization of $\varepsilon-\mathrm{Fe}_{2} \mathrm{O}_{3}$, J. Solid State Chem. 139, 93 (1998).

[9] Rustum Roy, V. G. Hill, and E. F. Osborn, Polymorphism of $\mathrm{Ga}_{2} \mathrm{O}_{3}$ and the system $\mathrm{Ga}_{2} \mathrm{O}_{3}-\mathrm{H}_{2} \mathrm{O}$, J. Am. Chem. Soc. 74, 719 (1952).

[10] N. Wang, F. S. Wen, L. Li, Y. F. Lü, S. C. Liu, Y. F. Lu, Z. Y. Liu, B. Xu, J. L. He, D. L. Yu, and Y. J. Tian, Magnetic frustration effect in polycrystalline $\mathrm{Ga}_{2-x} \mathrm{Fe}_{x} \mathrm{O}_{3}$, J. Magn. Magn. Mater. 322, 3595 (2010).

[11] M. Hatnean, J. Robert, M. T. Fernandez Diaz, E. Ressouche, A. Cousson, L. Pinsard-Gaudart, and S. Petit, Neutron scattering study of the magnetoelectric compound $\mathrm{GaFeO}_{3}$, Eur. Phys. J. Spec. Top. 213, 69 (2012).

[12] Daniel Stoeffler, First principles study of the electric polarization and of its switching in the multiferroic $\mathrm{GaFeO}_{3}$ system, J. Phys.: Condens. Matter 24, 185502 (2012).

[13] Daniel Stoeffler, First principles study of the spontaneous electric polarization in $\mathrm{Ga}_{2-x} \mathrm{Fe}_{x} \mathrm{O}_{3}$, Thin Solid Films 93, 4 (2013).

[14] J.-Y. Kim, T. Koo, and J.-H. Park, Orbital and Bonding Anisotropy in a Half-Filled $\mathrm{GaFeO}_{3}$ Magnetoelectric Ferrimagnet, Phys. Rev. Lett. 96, 047205 (2006).

[15] F. Ibrahim and M. Alouani, Impact of excess iron on the calculated electronic and magnetic properties of gallium ferrite, Phys. Rev. B 85, 174411 (2012).

[16] G. Kresse and J. Hafner, Ab initio molecular dynamics for liquid metals, Phys. Rev. B 47, 558 (1993).

[17] G. Kresse and J. Hafner, Ab initio molecular-dynamics simulation of the liquid-metal-amorphous-semiconductor transition in germanium, Phys. Rev. B 49, 14251 (1994).

[18] G. Kresse and J. Hafner, Norm-conserving and ultrasoft pseudopotentials for first-row and transition elements, J. Phys.: Condens. Matter 6, 8245 (1994).

[19] G. Kresse and J. Furthmüller, Efficiency of ab initio total energy calculations for metals and semiconductors using a plane-wave basis set, Comput. Mater. Sci. 6, 15 (1996).

[20] G. Kresse and J. Furthmüller, Efficient iterative schemes for $a b$ initio total-energy calculations using a plane-wave basis set, Phys. Rev. B 54, 11169 (1996).

[21] G. Kresse and D. Joubert, From ultrasoft pseudopotentials to the projector augmented-wave method, Phys. Rev. B 59, 1758 (1999).

[22] P. E. Blöchl, Projector augmented-wave method, Phys. Rev. B 50, 17953 (1994).
[23] S. L. Dudarev, G. A. Botton, S. Y. Savrasov, C. J. Humphreys, and A. P. Sutton, Electron-energy-loss spectra and the structural stability of nickel oxide: An LSDA+U study, Phys. Rev. B 57, 1505 (1998).

[24] Jochen Heyd, Gustavo E. Scuseria, and Matthias Ernzerhof, Hybrid functionals based on a screened Coulomb potential, J. Chem. Phys. 118, 8207 (2003).

[25] Jochen Heyd and Gustavo E. Scuseria, Efficient hybrid density functional calculations in solids: Assessment of the Heyd-Scuseria-Ernzerhof screened Coulomb hybrid functional, J. Chem. Phys. 121, 1187 (2004).

[26] Jochen Heyd, Gustavo E. Scuseria, and Matthias Ernzerhof, Hybrid functionals based on a screened Coulomb potential, J. Chem. Phys. 124, 219906 (2006).

[27] Aliaksandr V. Krukau, Oleg A. Vydrov, Artur F. Izmaylov, and Gustavo E. Scuseria, Influence of the exchange screening parameter on the performance of screened hybrid functionals, J. Chem. Phys. 125, 224106 (2006).

[28] See Supplemental Material at http://link.aps.org/supplemental/ 10.1103/PhysRevB.92.104408 for the within the HSE approximation relaxed GFO crystal structure (SM1).

[29] Z. H. Sun, S. Dai, Y. L. Zhou, L. Z. Cao, and Z. H. Chen, Elaboration and optical properties of $\mathrm{GaFeO}_{3}$ thin films, Thin Solid Films 516, 7433 (2008).

[30] R. V. Pisarev, The optical absorption spectrum is presented in $\mathrm{Pb}\left(\mathrm{Fe}_{0.5} \mathrm{Nb}_{0.5}\right) \mathrm{O}_{3}$ and $\mathrm{GaFeO}_{3}$, Sov. Phys. Solid State 7, 158 (1965).

[31] P. W. Anderson, Antiferromagnetism: Theory of superexchange interaction, Phys. Rev. 79, 350 (1950).

[32] John B. Goodenough, Theory of the role of covalence in the perovskite-type manganites $[\mathrm{La}, \mathrm{M}(\mathrm{ii})] \mathrm{MnO}_{3}$, Phys. Rev. 100, 564 (1955).

[33] John B. Goodenough, An interpretation of the magnetic properties of the perovskite-type mixed crystals $\mathrm{La}_{1-x} \mathrm{Sr}_{x} \mathrm{CoO}_{3-\lambda}$, J. Phys. Chem. Solids 6, 287 (1958).

[34] Junjiro Kanamori, Superexchange interaction and symmetry properties of electron orbitals, J. Phys. Chem. Solids 10, 87 (1959).

[35] J. B. Goodenough, Goodenough-Kanamori rule, Scholarpedia 3, 7382 (2008).

[36] In contrast to experimental values with an average doping concentration over the whole sample our substitutions are not strictly random but show long range order due to the periodicity of the super cell.

[37] Amritendu Roy, Somdutta Mukherjee, Surajit Sarkar, Sushil Auluck, Rajendra Prasad, Rajeev Gupta, and Ashish Garg, Effects of site disorder, off-stoichiometry and epitaxial strain on the optical properties of magnetoelectric gallium ferrite, J. Phys.: Condens. Matter 24, 435501 (2012).

[38] Amritendu Roy, Ashish Garg, Rajendra Prasad, and Sushil Auluck, A First-Principles study of structure-property correlation and the origin of ferrimagnetism in gallium ferrite, Adv. Mater. Phys. Chem. 02, 1 (2012).

[39] Chris G. Van de Walle and Jörg Neugebauer, First-principles calculations for defects and impurities: Applications to IIInitrides, J. Appl. Phys. 95, 3851 (2004).

[40] See Supplemental Material at http://link.aps.org/supplemental/ 10.1103/PhysRevB.92.104408 for further information on the strain induced geometry changes in stoichiometric GFO (SM2). 